\title{
Attraction Between Like-Charge Surfaces in Polar Mixtures
}

\author{
S. SAMIN ${ }^{1}$ and Y. TsOrI ${ }^{1}$ \\ 1 Department of Chemical Engineering and The Ilse Katz Institute for Nanoscale Science and Technology, Ben-Gurion \\ University of the Negev, 84105 Beer-Sheva, Israel.
}

PACS 64.70. Ja - Liquid-liquid transitions

PACS 64.75.Xc - Phase separation and segregation in colloidal systems

PACS 64.75.Jk - Phase separation and segregation in nanoscale systems

\begin{abstract}
We examine the force between two charged surfaces immersed in aqueous mixtures having a coexistence curve. For a homogeneous water-poor phase, as the distance between the surfaces is decreased, a water-rich phase condenses at a distance $D_{t}$ in the range $1-100 \mathrm{~nm}$. At this distance the osmotic pressure can become negative leading to a long-range attraction between the surfaces. The osmotic pressure vanishes at a distance $D_{e}<D_{t}$, representing a very deep metastable or globally stable energetic state. We give analytical and numerical results for $D_{t}$ and $D_{e}$ on the Poisson-Boltzmann level.
\end{abstract}

The forces between charged objects in electrolyte solutions are of fundamental importance in biology and colloidal science. Within the Poisson-Boltzmann (PB) mean-field theory, in a single pure solvent, the interaction between symmetrically charged colloids is always repulsive [1. Experiments, on the other hand, have shown that highly charged colloids can attract each other when multivalent ions are present. This discrepancy has been explained theoretically by counterion correlations [2], fluctuation-induced forces [3] and other non-electrostatic interactions [4]. More recently, the PB theory was generalized by strong coupling theory [5, 6, which predicts an attraction for large values of the so-called coupling parameter $7,8$.

In this Letter we use the PB theory to show that strong attractive forces appear between similarly charged colloids in mixtures. In mixtures one must also take into account that the medium itself becomes inhomogeneous due to dielectrophoretic and solvation-related forces. The preferential solvation energy of ions in one of the solvents [1,9] is appreciable and can even be much larger than the thermal energy 10 12. For a recent review on ion-specific solvation effects within the PB theory see [13. Previous works on preferential solvation in binary mixtures looked at the phase behavior in the bulk 14, 15], surface tension [16], and the interaction between surfaces but not in immiscible liquids [17. As is shown below, in partially miscible mixtures, the behavior is qualitatively different and strong forces occur. In colloidal suspensions these forces can have an important role not studied before 1822 .

We consider two positively charged colloids in a mixture of polar solvents. The colloids are modeled as flat surfaces located at $z= \pm D / 2$ and uniformly charged with a charge density $e \sigma$ per unit area, where $e$ is the elementary charge. A small amount of monovalent ions and weakly charged surfaces are assumed and therefore the coupling parameter is small and the PB theory is applicable 6. The partially miscible solvents have a coexistence curve below the mixture critical temperature $T_{c}$ in the absence of ions. This coexistence curve is further modified by the presence of ions [15].

The grand potential density is given by 14,23

$$
\begin{aligned}
\frac{\omega}{T} & =f_{b}(\phi)+\frac{C}{2}|\nabla \phi|^{2}+\frac{1}{2 T} \varepsilon(\phi)(\nabla \psi)^{2} \\
& +n^{+}\left(\log \left(v_{0} n^{+}\right)-1\right)+n^{-}\left(\log \left(v_{0} n^{-}\right)-1\right) \\
& -\left(\Delta u^{+} n^{+}+\Delta u^{-} n^{-}\right) \phi-\lambda^{+} n^{+}-\lambda^{-} n^{-}-\mu \phi
\end{aligned}
$$

Here the Boltzmann constant is set to unity, $T$ is the thermal energy and $C$ is a positive constant. $\phi$ is the volume fraction of water $(0 \leq \phi \leq 1)$; far away from the surfaces the mixture is homogeneous and water-poor with composition $\phi_{0}<1 / 2$ and ion densities $n_{0}$. We use a regular solution form for the free energy of a binary mixture $v_{0} f_{b}=\phi \log (\phi)+(1-\phi) \log (1-\phi)+\chi \phi(1-\phi)$ [24], where $\chi \sim 1 / T$ is the Flory parameter and $v_{0}=a^{3}$ is the molecular volume of both liquids. The third term in Eq. (1) is the electrostatic energy of the mixture, where $\psi$ is the electrostatic potential. The mixture dielectric 
constant depends on the composition through a linear relation: $\varepsilon(\phi)=\varepsilon_{c}+\left(\varepsilon_{w}-\varepsilon_{c}\right) \phi$, where $\varepsilon_{c}$ and $\varepsilon_{w}$ are the pure cosolvent and water dielectric constants, respectively. The second line of Eq. (1) is the ideal gas entropy of point-like ions, where $n^{+}$and $n^{-}$are the positive and negative ion density, respectively. The first term on the third line is the bilinear solubility interaction of the ions and the solvent: the parameters $\Delta u^{+}$and $\Delta u^{-}$measure the affinity of the positive and negative ions toward the water environment, respectively. We use the common case where both ions are hydrophilic and assume the symmetric interaction $\Delta u^{+}=\Delta u^{-}=\Delta u>0$. Lastly, $\lambda^{ \pm}$and $\mu$ are the Lagrange multipliers (chemical potentials) of the positive and negative ions and water composition, respectively.

The ion densities obey the Boltzmann distribution

$$
n^{ \pm}=v_{0}^{-1} \mathrm{e}^{\lambda^{ \pm}} \mathrm{e}^{\mp e \psi / T+\Delta u \phi}
$$

In a salt reservoir we have $\lambda^{ \pm}=\log \left(v_{0} n_{0}\right)-\Delta u \phi_{0}$. Alternatively, when the mixture contains only negative counterions, $\lambda^{-}$is determined self-consistently by charge conservation: $\int n^{-} \mathrm{d} z=2 \sigma$.

The electrostatic potential is determined by the Poisson equation

$$
\nabla \cdot(\varepsilon(\phi) \nabla \psi)=-e\left(n^{+}-n^{-}\right)
$$

supplemented by the boundary condition on the surfaces $-\mathbf{n} \cdot \nabla \psi( \pm D / 2)=e \sigma / \varepsilon(\phi)$, where $\mathbf{n}$ is the outward unit normal to the surface. Finally, the Euler-Lagrange equation for $\phi$ reads

$$
C \nabla^{2} \phi=\frac{\partial f_{b}}{\partial \phi}-\frac{1}{2 T} \frac{d \varepsilon}{d \phi}(\nabla \psi)^{2}-\Delta u\left(n^{+}+n^{-}\right)-\mu
$$

In order to isolate solvation and electrostatic effects, we assume zero short-range chemical or long-range van der Waals interactions with the surfaces, leading to the boundary condition $-\mathbf{n} \cdot \nabla \phi(z= \pm D / 2)=0$.

The net force exerted on the surfaces by the liquid is given by the osmotic pressure $\Pi=P_{z z}-P_{b}$, where $P_{b}$ is the bulk pressure: $P_{b} / T=\phi_{0} \partial f_{b}\left(\phi_{0}\right) / \partial \phi-f_{b}\left(\phi_{0}\right)+$ $2 n_{0}\left(1-\Delta u \phi_{0}\right) . \quad-P_{z z}$ is $z z$ component of the Maxwell stress tensor 17,25:

$$
\begin{aligned}
& \frac{P_{z z}}{T}=\frac{C}{2}\left(\frac{d \phi}{d z}\right)^{2}-C \phi \frac{d^{2} \phi}{d z^{2}}+\phi \frac{\partial f_{b}}{\partial \phi}-f_{b} \\
& +(1-\Delta u \phi)\left(n^{+}+n^{-}\right)-\frac{1}{2 T}\left(\phi \frac{d \varepsilon}{d \phi}+\varepsilon\right)\left(\frac{d \psi}{d z}\right)^{2}
\end{aligned}
$$

$P_{z z}$ is independent of $z$ and can be calculated at the midplane $(z=0)$ where by symmetry $d \phi / d z=d \psi / d z=0$. Multiplying Eq. (4) by $\phi$ and inserting into Eq. (5) we obtain:

$$
\Pi=T n_{m}-T \omega_{b}\left(\phi_{m}\right)-P_{b}
$$

where $\omega_{b}=f_{b}-\mu \phi$ and $\phi_{m}$ and $n_{m}$ are the composition and total ion density at the midplane, respectively.
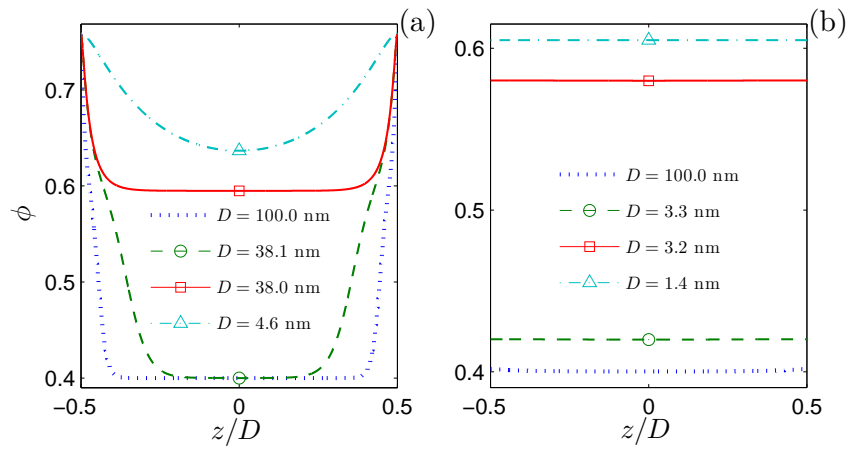

Fig. 1: (a) Composition profiles $\phi(z)$ in the strong screening limit for $D<D_{t}$ (dotted line), just before and after $D_{t}$ (dashed and solid lines) and at $D_{e}$ (dash-dot line). Here $n_{0}=0.1 \mathrm{M}$, $\Delta u=2$ and $\sigma=1 \mathrm{~nm}^{-2}$. (b) The same in the ideal gas regime with no salt, $\Delta u=11$ and $\sigma=0.01 \mathrm{~nm}^{-2}$. Here and, unless stated otherwise, in all other figures we took for the mixture $\phi_{0}=0.4, T / T_{c}=0.9915$ and $C=\chi / a$ 24]. As an approximation of a water-1-butanol mixture we used $T_{c}=398 \mathrm{~K}$, $v_{0}=3 \times 10^{-29} \mathrm{~m}^{3}, \varepsilon_{a}=17.8$ and $\varepsilon_{w}=80$.

In aqueous mixtures, water is drawn towards the walls by field gradients (dielectrophoretic) and solvent-induced (electrophoretic) forces 17,23 . These forces are interdependent as is evident from Eqs. (3) and (4). From the solution of the governing equations we find two possible scenarios, distinguished according to the composition profiles at infinite separation. In the first scenario, when the surfaces are far apart, $\phi(z)$ near each surface is composed of three layers: a thin water-rich layer $(\phi>1 / 2)$ of width $t$ at the surfaces, a second layer with width of the bulk correlation length $\xi$ adjacent to it, where the composition $\phi \gtrsim \phi_{0}$ decays to the bulk value, and a third "bulk" layer where $\phi \approx \phi_{0}$.

As the surface separation decreases down to $D=D_{t}$, the general features of this profile do not change. In particular, only the width of the $\phi \approx \phi_{0}$ region changes if screening is strong, that is, when $l_{D} \ll D$ where $l_{D} \sim n_{0}^{-1 / 2}$ is the Debye length calculated at $\varepsilon=\varepsilon\left(\phi_{0}\right)$. The dashed curve in Fig. 1 (a) shows $\phi(z)$ just before $D_{t}$ for this case.

In the second scenario, dielectrophoretic and solvation forces are not strong enough and at large surface separations $\phi$ is always close to the bulk value $\phi_{0}$. As $D$ decreases from infinity the water composition between the surfaces increases continuously. In particular, in the ideal gas regime, defined by $b \gg D$, where $b \propto \sigma^{-1}$ is the GouyChapman length, $\phi(z)$ is nearly uniform. Fig. 1(b) shows such profiles for a mixture containing only counterions. Here, the ion profile $n(z)$ and electric field are nearly uniform leading to small composition gradients.

In both scenarios, when $D<D_{t}$ the whole space between the surfaces becomes rich in water (see the solid curves in Fig. 11. The transition occurs at a distance in the range $D_{t}=1-100 \mathrm{~nm}$ depending on the parameter values, and is accompanied by a decrease in the osmotic 

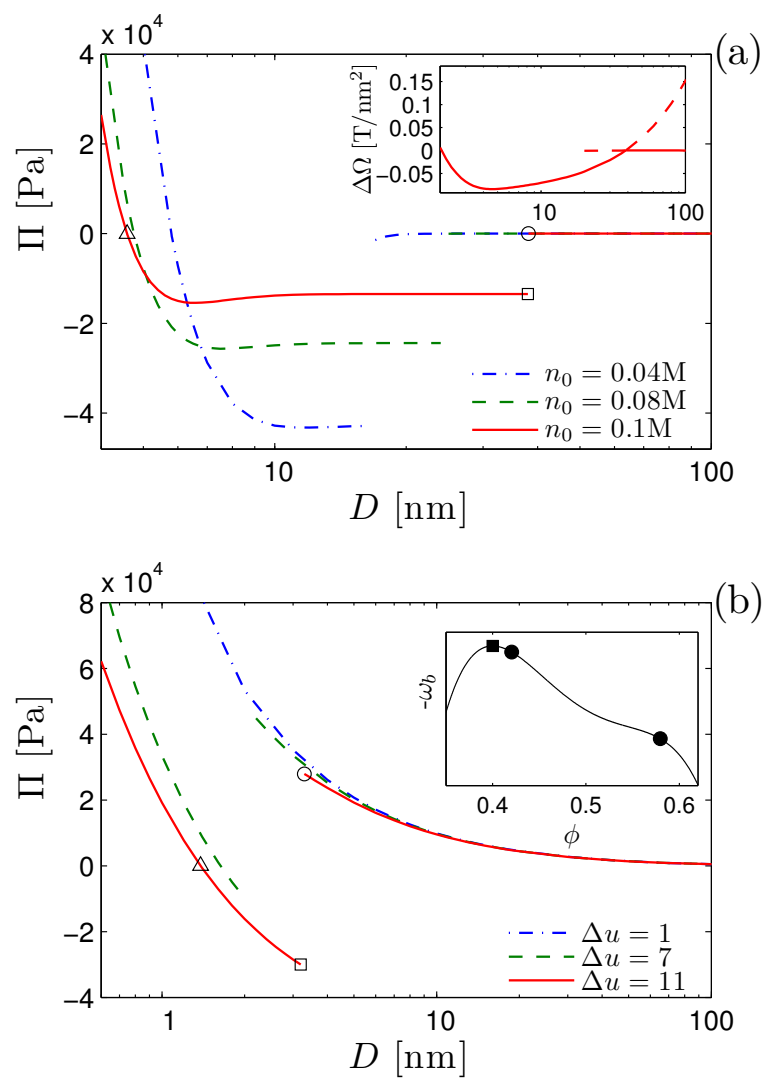

Fig. 2: (a) Osmotic pressure $\Pi$ vs surface separation $D$ in the strong screening limit for different values of $n_{0}$ [other parameters as in Fig. [1(a)]. Inset: excess grand potential for $n_{0}=0.1 \mathrm{M}$. solid (dashed) line corresponds to stable (metastable) solutions. (b) $\Pi$ in the ideal gas regime for different values of $\Delta u$ [other parameters as in Fig. 11(b)]. Inset: the function $-\omega_{b}(\phi)$. Filled square and circle markers correspond to $\phi_{0}$ and the binodal compositions, respectively. In (a) and (b) open markers on solid lines correspond to distances $D$ with the same symbols in Fig. 1] (a) and (b).

pressure. This can lead to a negative osmotic pressure, such that a surface separation $D_{e}<D_{t}$ exists at which $\Pi=0 . \phi(z)$ at $D=D_{e}$ is shown in the dash-dot curves in Fig. 1.

The excess grand potential per unit area relative to infinite separation is $\Delta \Omega(D)=-\int_{\infty}^{D} \Pi\left(D^{\prime}\right) \mathrm{d} D^{\prime} . \Delta \Omega(D)$ has a cusp at $D=D_{t}$ and this results in a discontinuity of the pressure. Close to $D_{t}$, both a water-poor and a waterrich profiles are solutions of the Euler-Lagrange equations but only one of these is a stable solution. At $D>D_{t}$ the water-poor phase is stable, at $D<D_{t}$ the water-rich phase is stable, and at $D=D_{t}$ the grand potentials of the two phases are equal. Furthermore, $\Delta \Omega$ has a minimum at $D_{e}\left(D_{e}<D_{t}\right)$ corresponding to mechanical equilibrium. An example of $\Delta \Omega(D)$ is shown in the inset of Fig. 2(a), where the dashed line is $\Delta \Omega$ of metastable solutions.

The transition to a water-rich phase has a different phys-

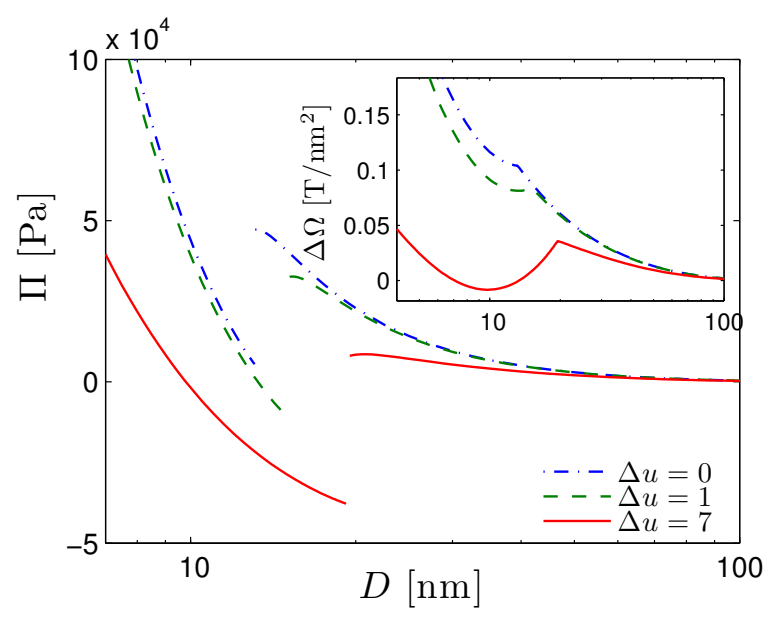

Fig. 3: Osmotic pressure in the weak screening regime with $n_{0}=10^{-4} \mathrm{M}$ and $\sigma=1 \mathrm{~nm}^{-2}$ for three values of $\Delta u$. Inset: the corresponding excess grand potential $\Delta \Omega$.

ical origin in the two limiting cases. In the strong screening regime, the transition is promoted by the energy gained when the interface vanishes, as in capillary condensation. The thin water-rich layer near the walls remains nearly independent on $D$. In the ideal gas regime, on the other hand, when the composition approaches the coexistence composition, preferential solvation promotes a transition to a water-rich phase. For intermediate cases, both mechanisms play a role in the transition. These effects are enhanced but are not limited to the vicinity of the critical temperature where the differences between the phases become smaller.

In Fig. 2 we plot the osmotic pressure as a function of surface spacing; when $\Pi>0$ the surfaces repel each other while for $\Pi<0$ they attract. $\Pi$ is discontinuous at $D=D_{t}$. In Fig. 2(a) we show $\Pi$ for different values of $n_{0}$ in the strong screening limit. An increase in $n_{0}$ decreases $\Pi$ at large distances but increases it at small distances (entropy loss of the ions).

The negative jump in $\Pi$ at $D=D_{t}$ increases with decreasing $n_{0}$. This can be explained by the interplay between the first two terms in Eq. (6). At $D=D_{t}$ the increase in $\phi_{m}$ dominates the decrease in $\Pi$, an effect more pronounced for smaller values of $n_{0}$ since the positive ideal gas term is proportional to $n_{m} \propto n_{0}$. Recall that in a pure solvent only the ideal gas term exists and hence $\Pi$ is always positive. Furthermore, this entropic repulsion will eventually dominate $\Pi$ leading to $\Pi=0$ at a separation $D_{e}$.

In Fig. 2(b) we plot $\Pi$ for different values of $\Delta u$ in the ideal gas regime. For $\Delta u=1$ the pressure is purely repulsive since preferential solvation is not strong enough to induce a water-rich phase. For $\Delta u=7$ the interaction is repulsive down to $D_{t}$ where $\Pi$ becomes negative. When $\Delta u$ increases to 11 the transition is at a larger distance and to a more negative pressure. The inset of Fig. 2(b) 
shows the function $-\omega_{b}(\phi)$, showing a decrease in pressure at the transition [cf. Eq. (6)].

Fig. 3 shows $\Pi$ in the weak screening regime where $l_{D} \approx$ $D$ and for three different values of $\Delta u$. When $\Delta u=0$, the dielectrophoretic force alone can induce a water-rich phase albeit the pressure is always repulsive. The pressure can become attractive for $\Delta u=1$ or 7 . Corresponding curves of $\Delta \Omega$ are plotted in the inset of Fig. 3. These show a metastable minimum at a finite value of $D, D=D_{e}$, for $\Delta u=1$ and a global minimum for $\Delta u=7$. The depth of the minimum for $\Delta u=7$ is $\approx 440 T$ for two colloids with an effective surface area of $100 \mathrm{~nm} \times 100 \mathrm{~nm}$.

For $\Delta u=10$, a second metastable minimum can appear in the curve $\Delta \Omega(D)$ at a smaller value of $D$, see the dashed and dash-dot curves in Fig. 4. A similar value of $\Delta u$ is cited in the literature for mixtures of water and 1butanol containing $\mathrm{NaCl}$ at room temperature 12 . When $\sigma$ is further increased (solid curves), this minimum can become globally stable. For large enough $\sigma$, only the second minimum exists (not shown). In this large $\Delta u$ case, preferential solvation leads to the liquid between the surfaces being nearly pure water; $\phi_{m}$ is close to unity and $\omega_{b}$ diverges.

The distance $D_{t}$ can be obtained in the two limiting cases presented in Fig. 1. In the strong screening limit we use continuity of $\Delta \Omega$ at $D_{t}$ to obtain [26]:

$$
D_{t} \simeq 2 t+2 \frac{\xi P_{b}+\int_{D / 2-\xi-t}^{D / 2-t} \omega(\phi(z)) \mathrm{d} z}{P_{b}-P_{h}}
$$

$P_{h}$, the pressure in the water-rich phase, is calculated from $P_{h}=T\left(n_{h}^{+}+n_{h}^{-}\right)-T \omega_{b}\left(\phi_{h}\right)+P_{b}$, where $n_{h}^{ \pm}=$ $n_{0} \mathrm{e}^{\Delta u\left(\phi_{h}-\phi_{0}\right)}$ are the midplane values and $\phi_{h}$ is obtained from

$$
\frac{\partial f_{b}}{\partial \phi}\left(\phi_{h}\right)-2 \Delta u n_{0} \mathrm{e}^{\Delta u\left(\phi_{h}-\phi_{0}\right)}-\mu_{0}=0
$$

where we used $\psi \approx 0$ at the midplane.

At $D=D_{t}$ the surfaces can be regarded as isolated and a zeroth-order approximation of $\psi$ can be obtained using the well known result for a single surface 1 with a homogeneous $\phi \approx \phi_{h}$ and a modified Debye length $\left(\varepsilon\left(\phi_{h}\right) T /\left(2 e^{2} n_{0} \mathrm{e}^{\Delta u\left(\phi_{h}-\phi_{0}\right)}\right)\right)^{1 / 2}$. Since at $|z|<D / 2-t$ $e \psi / T \ll \Delta u\left(\phi_{h}-\phi_{0}\right), t$ is obtained from the condition $e \psi / T=0.01 \Delta u\left(\phi_{h}-\phi_{0}\right)$. In analogy with classical mean field theory [24], the Landau expansion of Eq. (8) around $\phi_{c}=1 / 2$ gives for $\xi$

$$
\xi \approx v_{0}^{1 / 3} \frac{1}{\sqrt{1-\frac{T}{T_{c}}-2 \Delta u v_{0} n_{0} \mathrm{e}^{\Delta u\left(\frac{1}{2}-\phi_{0}\right)}\left(1+\frac{\Delta u}{4}\right)}}
$$

The preferential solvation term in the root is comparable in magnitude to $1-T / T_{c}$. Thus, the bulk correlation length is modified appreciably by the preferential solvation of the ions.

Fig. 5(a) shows the comparison of $D_{t}$ from Eq. (7) with numerical results for different values of $n_{0}$. The agreement
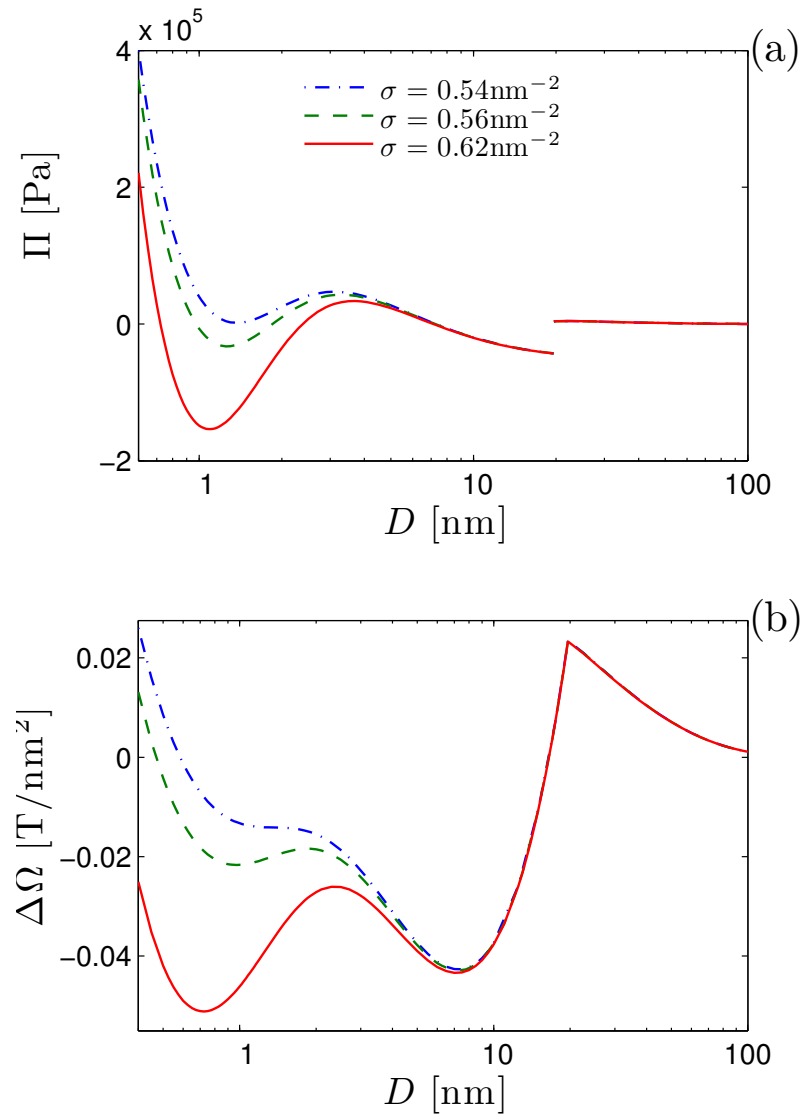

Fig. 4: (a) osmotic pressure for a large $\Delta u=10, n_{0}=10^{-4} \mathrm{M}$ and three values of $\sigma$. (b) the corresponding excess grand potential. For $\sigma=0.54 \mathrm{~nm}^{-2}, \Delta \Omega$ has only one minimum (dashdot curve) at $D<D_{t}$. A second, metastable minimum appears for $\sigma=0.56 \mathrm{~nm}^{-2}$ (dashed curve), whereas for $\sigma=0.62 \mathrm{~nm}^{-2}$ (solid curve) this minimum becomes globally stable.
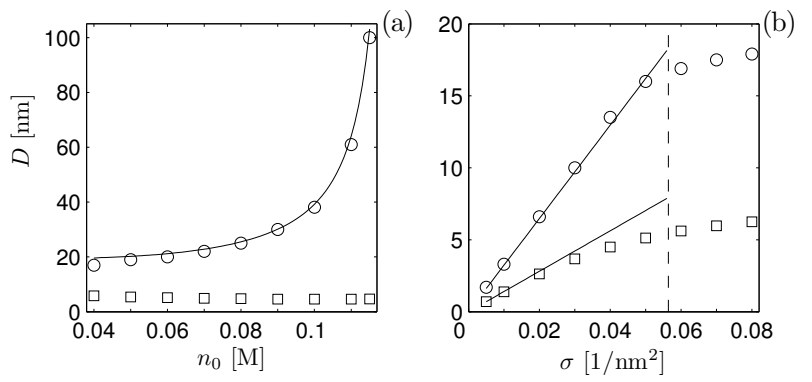

Fig. 5: Transition and equilibrium separations $D_{t}$ (circles) and $D_{e}$ (squares). (a) The strong screening case vs $n_{0}$. (b) The ideal gas regime vs $\sigma$. Solid curves are analytical expressions given in the text. In (a) we approximated $\phi(z)$ by a linear decrease from $\phi_{h}$ to $\phi_{0}$ at the interface region. In (b), for $\sigma$ beyond the dashed curve, a water-rich layer near the surfaces exists even as $D \rightarrow \infty$.

is quite good despite the crude approximation. $D_{e}$ in Fig. 5 (a) decreases slightly when $n_{0}$ increases, its value being $\approx 5 \mathrm{~nm}$.

In the ideal gas regime $\phi(z) \approx$ const. and the counter 


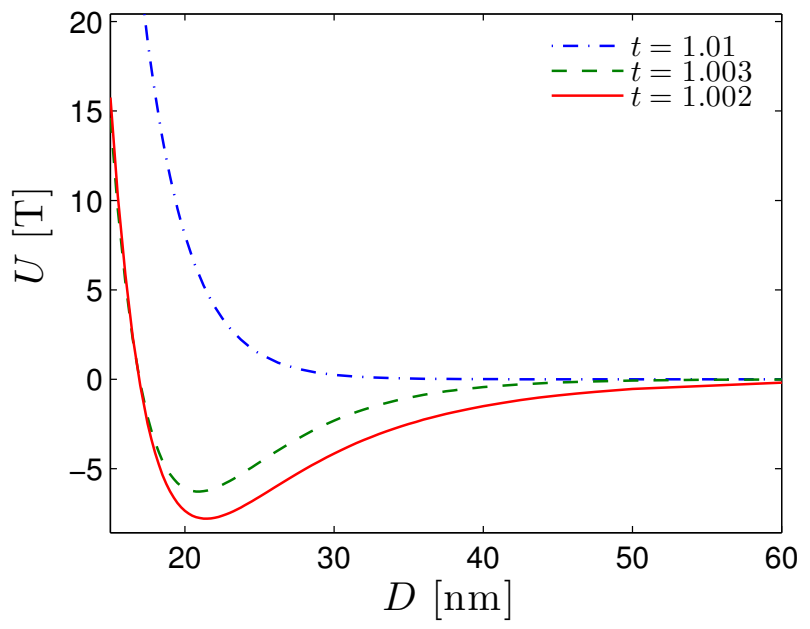

Fig. 6: Interaction potential for a mixture with a critical composition $\phi_{0}=1 / 2$ at three temperatures above $T_{c}$. For $t=1.01$ (dash-dot curve) the interaction is purely repulsive. When the temperature is decreased the interaction turns attractive (dashed curve), becoming stronger closer to $T_{c}$ (solid curve). Here $n_{0}=0.01 \mathrm{M}, \Delta u=4$ and $\sigma=1 \mathrm{~nm}^{-2}$. The walls have a surface of $A=0.01 \mu \mathrm{m}^{2}$.

ions density $n^{-}$is uniform and equal to the average charge density $24:\left\langle n^{-}\right\rangle \simeq 2 \sigma / D$. The composition equation reads

$$
\frac{\partial f_{b}}{\partial \phi}-\frac{2 \Delta u \sigma}{D}-\mu_{0}=0
$$

Here preferential solvation merely shifts the chemical potential of the mixture. Hence, $D_{t}$ occurs when $\phi$ is the binodal composition at which $\partial f_{b} / \partial \phi=0$. Thus,

$$
D_{t} \simeq-2 \sigma \Delta u / \mu_{0}
$$

which means that $D_{t} \rightarrow \infty$ when the bulk composition approaches the binodal $\left(\mu_{0} \rightarrow 0\right)$.

$D_{e}$ can be found by noting that $\Pi=0$ in Eq. (6) gives $n_{e}=\omega_{b}\left(\phi_{e}\right)+P_{b} / T$, where $\phi_{e}$ and $n_{e}$ are the composition and ion density at $D_{e}$, respectively. Inserting this into Eq. (4) we obtain for $\phi_{e}$

$$
\frac{\partial \omega_{b}}{\partial \phi}\left(\phi_{e}\right)=\Delta u\left(\omega_{b}\left(\phi_{e}\right)+P_{b} / T\right)
$$

which is solved and then used to get $n_{e}$ and $D_{e}$ from the equations for $\Pi$ and $\left\langle n^{-}\right\rangle$, respectively. Comparison of the formulae for $D_{t}$ and $D_{e}$ with the full numerical results are presented in Fig. 5(b). As expected, the agreement is good for small values of $\sigma$ where the ideal gas limit is valid. The dashed curve in Fig. 5(b) marks the charge density above which a water-rich layer exists at infinite separation and the above approximations no longer hold.

We find that at an attractive interaction is also possible at $T>T_{c}$. In this case, since there is no miscibility gap above $T_{c}$, the water-reach transition is missing and hence no discontinuity in the pressure is observed. Instead the pressure becomes smoothly attractive as the plate distance is reduced. Fig. 6 shows the interaction potential $U=\Delta \Omega \times A$ for two plates in a critical mixture at three different temperatures. As the critical temperature is approached, a purely repulsive potential (dash-dot curve) becomes attractive (dashed curve). The interaction is more attractive closer to $T_{c}$ (solid curve). The interaction energy and length scales and the temperature trend shown in Fig. 6 are similar to those observed in recent experiments on the salt-dependent interaction of a charged particle suspended in a critical binary liquid mixture near a charged wall 21]. In light of this, we believe that the mechanism we describe may be important to capture correctly electrostatic effects in these experiments. Thus, it is possible to attribute some of the effects shown in these experiments to solvation related forces 27 in addition to the critical Casimir forces.

The qualitative difference in the interaction between charged colloids or macromolecules in mixtures compared to pure solvents stems from the contribution to $\Pi$ of the second term in Eq. (6): $\omega_{b}\left(\phi_{m}\right)$ - the midplane mixture grand potential. This term is absent in pure solvents. Thus, unlike similar ion induced phase transitions in pure solvents [28] we observe a jump to a negative pressure (at $\left.D_{t} \approx 1-100 \mathrm{~nm}\right)$. Moreover, the nontrivial dependence of $\omega_{b}\left(\phi_{m}\right)$ on the system parameters $\left(T, \phi_{0}, n_{0}, \sigma\right)$ through the governing equations leads to qualitatively different behavior compared to usual condensation transitions due to surface fields [26]. In addition the attractive force is in many cases strong and long-range compared to the van der Waals force 1 as is evident by the values of $\Pi$ at $D=D_{t}$ [Fig. 2 and Fig. 3 and the energy minimum being deeper than $\sim 100 T$.

The mechanism we describe should be at play in the aggregation of charged colloids in mixtures near the coexistence temperature 18, 19. We believe it is directly relevant to the attraction seen between colloids and surfaces in mixtures also far from $T_{c}$ and attributed to critical Casimir forces 2022 .

$$
* * *
$$

We gratefully acknowledge numerous discussions with D. Andelman, C. Bechinger, H. Diamant, J. Dietrich, L. Helden, O. Nelen, A. Onuki, P. Pincus, R. Podgornik, S. Safran and M. Schick. This work was supported by the Israel Science Foundation under grant No. 11/10 and the European Research Council "Starting Grant" No. 259205.

\section{REFERENCES}

[1] Israelachvili J. N., Intermolecular and Surface Forces 2nd Edition (Academic Press, London) 1992.

[2] Kuellander R. and Marčelua S., Chem. Phys. Lett. , 127 (1986) 402 . 
[3] Lau A. W. C., Levine D. and Pincus P., Phys. Rev. Lett. , 84 (2000) 4116.

[4] Burak Y. and Andelman D., Phys. Rev. E , 62 (2000) 5296.

[5] Moreira A. G. and Netz R. R., Europhys. Lett., 52 (2000) 705.

[6] Naji A., Kanduč M., Netz R. R. and Podgornik R., Exotic electrostatics: Unusual features of electrostatic interactions between macroions in Understanding Soft Condensed Matter via Modeling and Computation, edited by Hu W.-B. and SHI A.-C., (World Scientific, Singapore) 2010.

[7] Netz R., Eur. Phys. J. E , 5 (2001) 557.

[8] Moreira A. G. and Netz R. R., Phys. Rev. Lett. , 87 (2001) 078301.

[9] Marcus Y., J. Chem. Soc., Faraday Trans. 1, 84 (1988) 1465.

[10] Osakai T. and Ebina K., J. Phys. Chem. B , 102 (1998) 5691.

[11] Hung L. Q., J. Electroanal. Chem. , 115 (1980) 159; 149 (1983) 1.

[12] Marcus Y., Pure Appl. Chem. , 55 (1983) 977.

[13] Ben-Yaakov D., Andelman D., Podgornik R. and Harries D., Curr. Opin. Colloid Interface Sci. , In Press (2011).

[14] Onuki A., Phys. Rev. E , 73 (2006) 021506.

[15] Окамото R. and Onuki A., Phys. Rev. E , 82 (2010) 051501.

[16] Onuki A., J. Chem. Phys., 128 (2008) 224704.

[17] Ben-YaAkov D., Andelman D., Harries D. and PodGornik R., J. Phys. Chem. B , 113 (2009) 6001.

[18] Beysens D. and Estève D., Phys. Rev. Lett. , 54 (1985) 2123.

[19] Law B. M., Petit J.-M. and Beysens D., Phys. Rev. E , 57 (1998) 5782.

[20] Hertlein C., Helden L., Gambassi A., Dietrich S. and Bechinger C., Nature, 451 (2008) 172.

[21] Nellen U., Dietrich J., Helden L., Chodankar S., Nygård K., van der Veen J. F. and Bechinger C., Soft Matter , 7 (2011) 5360.

[22] Bonn D., Otwinowski J., Sacanna S., Guo H., WegDAm G. and Schall P., Phys. Rev. Lett. , 103 (2009) 156101.

[23] Tsori Y. and Leibler L., Proc. Nat. Acad. Sci. , 104 (2007) 7348.

[24] Safran S., Statistical Thermodynamics of Surfaces, Interfaces, and Membranes (Westview Press, New York) 1994.

[25] Landau L. D., Lifshitz E. M. and Pitaevski L. P., Electrodynamics of Continuous Media 2nd Edition (Butterworth-Heinemann, Amsterdam) 1984.

[26] Evans R., Marconi U. M. B. and Tarazona P., J. Chem. Phys., 84 (1986) 2376.

[27] Bier M., Gambassi A., Oettel M. and Dietrich S., arXiv:1104.5703v1, (2011).

[28] Harries D., Podgornik R., Parsegian V. A., MarOr E. and Andelman D., J. Chem. Phys., 124 (2006) 224702 . 